\title{
Eventos extremos de precipitação no Alto Rio Grande, MG: Análise probabilística
}

\author{
José A. Junqueira Júnior ${ }^{1}$, Carlos R. de Mello² \& Geovane J. Alves ${ }^{3}$
}

\section{Palavras-chave:}

distribuições assintóticas

hidrologia estatística

inferência estatística

\begin{abstract}
R E S U M O
As distribuições de probabilidades de valores extremos desempenham importante papel na modelagem de eventos extremos. Elas têm sido amplamente aplicadas em estudos hidrológicos a fim de predizer, para um nível de confiança, valores esperados num dado tempo de retorno. Várias distribuições têm sido empregadas com diferentes métodos de ajuste. Objetivou-se neste trabalho verificar a aderência das distribuições GEV e Gumbel aos dados de precipitação diária máxima anual de doze estações pluviométricas no Alto Rio Grande, MG. Foram consideradas três sub-regiões com características fisiográficas peculiares, cada uma com quatro estações pluviométricas em seus domínios, Planalto Campos das Vertentes (PCV), Serra da Mantiqueira (SM) e uma sub-região de Transição (TR) entre as duas. Para a estimação dos parâmetros utilizou-se o método dos momentos, máxima verossimilhança e momentos-L. A aderência dos ajustes foi avaliada pelos testes de Filliben e Qui-quadrado $\left(\chi^{2}\right)$. A metodologia dos momentos-L mostrou-se como a melhor opção, independente da sub-região sendo que se recomenda, para as sub-regiões SM e TR, a distribuição Gumbel, enquanto para o PCV a melhor opção de ajuste é a GEV.
\end{abstract}

\section{Key words:}

asymptotic distributions

statistical hydrology

statistical inference

\section{Extreme rainfall events in the Upper Rio Grande, MG: Probabilistic analysis}

\begin{abstract}
A B S T R A C T
Probabilistic models have played an important role for stochastic modeling of the extreme events. They have been widely used in hydrological studies to predict, for a confidence level, expected values in a given return period. Several probabilistic models have been applied based on different adjustment methods which estimate the statistical moments of them. The aim of this study was to verify the adherence of Generalized Extreme Values (GEV) and Gumbel probabilistic models at two frequency of occurrence of heavy rainfall events in the Upper Rio Grande region (UGR), Southern Minas Gerais. UGR region was divided into three sub-regions with similar physiographic features in which four rain-gauging stations were studied: Plateau of Campos das Vertentes (PCV), Mantiqueira Range (SM) and a transition sub-region (TR). For estimating the distribution parameters, the Moment Method, Maximum Likelihood and L-moments were tested. The adherence of probabilistic models was evaluated by both Filiben and Chi-square tests. L-moments method showed to be the best method for all sub-regions and models. In addition, for the SM and TR subregions Gumbel model produced better results while for the PCV, GEV model was more appropriate.
\end{abstract}

Protocolo 040-2014 - 06/02/2014 • Aprovado em 21/11/2014 • Publicado em 02/03/2015

${ }^{1}$ Núcleo de Recursos Naturais/IF Sudeste MG. Bom Sucesso, MG. E-mail: jose.junqueira@ifsudestemg.edu.br (Autor correspondente)

${ }^{2}$ Departamento de Engenharia/UFLA. Lavras, MG. E-mail: crmello@deg.ufla.br

${ }^{3}$ Departamento de Engenharia/UFLA. Lavras, MG. E-mail: geovane@agricola.eng.br 


\section{INTRODUÇÃo}

O conhecimento da melhor metodologia de ajuste para distribuições de probabilidades é um passo imprescindível na redução da vulnerabilidade humana contra catástrofes naturais. A estimação da probabilidade de ocorrência de eventos extremos que são potencialmente nocivos à sociedade, como o caso da precipitação diária máxima anual (PDMA), é uma forte motivação para o estudo e modelagem da estatística desses eventos. Sendo a ocorrência dessas precipitações um processo aleatório (Hartmann et al., 2011), que não permite uma previsão determinística com grande antecedência, o ajustamento de um modelo probabilístico que melhor descreve o processo se faz necessário a fim de se fazer estimativas de eventos extremos.

Diversos trabalhos têm mostrado que as distribuições de probabilidade têm sido frequentemente utilizadas em estudos desta natureza (Takara \& Kobayashi, 2013) algumas delas, como o caso da distribuição generalizada de valores extremos (GEV), têm apresentado melhor desempenho em diversos resultados (Blain \& Camargo, 2012). Em contrapartida, outros estudos têm evidenciado que a distribuição Gumbel é a que melhor se ajusta aos fenômenos meteorológicos extremos, entre os quais a precipitação diária máxima anual (Hartmann et al., 2011).

Segundo Naghettini \& Pinto (2007), os parâmetros das distribuições de probabilidades devem ser estimados por algum procedimento da estatística matemática. De acordo com os autores, existe uma gama de métodos de estimação de parâmetros dentre os quais se destaca o método dos momentos (MM), da máxima verossimilhança (MV) e dos momentos-L (M-L). Desta forma, um problema que surge na prática é o da escolha do melhor método de ajuste e da distribuição de valores extremos mais adequada para determinada amostra de dados.

A região do Alto Rio Grande, localizada no Sul e Sudeste do Estado de Minas Gerais, é caracterizada por regiões fisiográficas de grande importância no contexto ambiental, especialmente no que tange à "produção de água" (Alvarenga et al., 2011). Segundo Mello et al. (2012) as duas sub-regiões mais representativas do Alto Rio Grande são a Serra da Mantiqueira (SM) e o Planalto Campos das Vertentes (PCV). Por meio de uma análise na literatura existente foi possível constatar que os trabalhos desenvolvidos para a referida região, como o de Ávila et al. (2009) não consideram, até então, as condições específicas de cada sub-região no ajuste e na seleção do melhor modelo probabilístico.

Este trabalho propôs verificar a adequacidade das metodologias MV, MM e M-L ajustadas às duas distribuições assintóticas empíricas, sendo a GEV e a Gumbel para máximos. Para tanto foram selecionadas doze séries históricas de precipitação diária máxima anual (PDMA), divididas em 3 sub-regiões do Alto Rio Grande: Planalto Campos das Vertentes (PCV), Serra da Mantiqueira (SM) e uma subregião de transição (TR).

\section{Material e Métodos}

Os dados de PDMA foram obtidos junto à Agência Nacional de Águas (ANA) provenientes de 12 estações pluviométricas. Como critério para a seleção das estações, foram selecionadas aquelas que se encontram sob os domínios das sub-regiões estudadas e que apresentam séries históricas com no mínimo 25 anos de observações (Tabela 1) além de não apresentarem falhas ou interrupções em seus registros.

Tabela 1. Estações pluviométricas, localização e período de aquisição de dados

\begin{tabular}{lcc}
\hline $\begin{array}{c}\text { Estação } \\
\text { pluviométrica }\end{array}$ & $\begin{array}{c}\text { Sub-região } \\
\text { Ibituruna }\end{array}$ & $\begin{array}{c}\text { Período de aquisição/ } \\
\text { Tamanho da série }\end{array}$ \\
\hline Ibertioga & PCV & 1968 a 2011 - 43 anos \\
Itumirim & PCV & 1941 a 2011 - 70 anos \\
\hline Macaia & PCV & 1941 a 2007 - 66 anos \\
Carrancas & PCV & 1969 a 2002 - 33 anos \\
Madre de Deus & TR & 1984 a 2012 - 28 anos \\
Fazenda Laranjeiras & TR & 1941 a 2006 - 65 anos \\
São Vicente & TR & 1972 a 2006 - 34 anos \\
Aiuruoca & TR & 1953 a 2011 - 58 anos \\
Alagoa & SM & 1945 a 2011- 66 anos \\
\hline Carvalhos & SM & 1942 a 2012 - 70 anos \\
Bom Jardim & SM & 1967 a 2011 - 44 anos \\
\hline
\end{tabular}

A função cumulativa de probabilidades (FCP) da distribuição Gumbel é dada por:

$$
\text { FCP }: P(x \leq x)=e^{-e^{-\alpha(x-\mu)}}
$$

em que:

$$
\begin{array}{ll}
\alpha & \text { - parâmetro de escala } \\
\mu & \text { - parâmetro de forma } \\
\mathrm{x} & \text { - valores de PDMA }
\end{array}
$$

A estimativa dos parâmetros desta distribuição por MM é a seguinte:

$$
\begin{gathered}
\operatorname{VAR}[X]=\frac{\pi^{2}}{\left(6 \times \alpha^{2}\right)}=\frac{1,645}{\alpha^{2}} \\
E[X]=\mu+\frac{0,577}{\alpha}
\end{gathered}
$$

Assim, substituindo a variância $\left(s^{2}\right)$ na Eq. 2 e a média na Eq. 3, tem-se:

$$
\begin{gathered}
\hat{\alpha}=\frac{1,2826}{S} \\
\hat{\mu}=\bar{X}-0,451 \times S
\end{gathered}
$$

O método MV consiste, basicamente, em maximizar a função densidade de probabilidade como função dos parâmetros da distribuição produzindo a probabilidade máxima de ocorrência das observações (Sharda et al., 2008). O equacionamento para a condição de máximo resulta em um sistema de igual número de equações e incógnitas cujas soluções produzem os estimadores de MV (Naghettini \& Pinto, 2007). Seguindo as recomendações de Mello et al. (2010) e se fazendo uma série de manipulações matemáticas, chegar-se-á às seguintes equações: 


$$
\begin{aligned}
& \frac{1}{\alpha}=\bar{X}-\frac{\sum_{i=1}^{n} x_{i} \exp \left(-\alpha \cdot x_{i}\right)}{\sum_{i=1}^{n} \exp \left(-\alpha \cdot x_{i}\right)} \\
& \exp (-\alpha \cdot \mu)=\frac{\sum_{i=1}^{n} e(-\alpha \cdot \mu)}{n}
\end{aligned}
$$

Como a Eq. 6 não tem solução explícita para $\alpha$, utilizou-se o método de Newton-Raphson. De posse do resultado de a aplica-se este valor à Eq. 7 calculando-se, então, o parâmetro de forma $\mu$.

Para a metodologia M-L os parâmetros da distribuição Gumbel foram estimados pelas seguintes equações (Hosking, 1990):

$$
\begin{gathered}
\hat{\alpha}=\frac{\ln (2)}{\lambda_{2}} \\
\hat{\mu}=\lambda_{1}-\frac{0,5772}{\hat{\alpha}}
\end{gathered}
$$

em que:

$\lambda_{1}$ e $\lambda_{2}$ - parâmetros de posição e de escala da distribuição, respectivamente, e são determinados pelas seguintes equações:

$$
\begin{gathered}
\lambda_{1}=\alpha_{0} \\
\lambda_{2}=\alpha_{0}-2 \alpha_{1}
\end{gathered}
$$

em que:

a - determinado com base em análises combinatórias pela Eq. 12, a saber:

$$
\hat{\alpha}_{s}=\frac{1}{N} \cdot \sum_{i=1}^{N} \frac{\left(\frac{N-i}{s}\right)}{\left(\frac{N-1}{s}\right)} \cdot x_{i}
$$

em que:

$$
\begin{array}{ll}
\mathrm{s} & \text { - número inteiro variando de } 0 \text { a } 3 \\
\mathrm{~N} & \text { - tamanho da amostra } \\
\mathrm{i} & \text { - ordem do valor } \mathrm{x}
\end{array}
$$

De acordo com Naghettini \& Pinto (2007), a GEV apresenta a seguinte expressão da função cumulativa da probabilidade:

$$
\mathrm{FCP}=\mathrm{P}(\mathrm{X} \geq \mathrm{xi})=\exp \left\{-\left[1-\xi \cdot\left(\frac{\mathrm{x}-\mu}{\alpha}\right)\right]^{\frac{1}{\xi}}\right\}
$$

em que:

a, $\xi$ e $\mu$ - parâmetros de escala, forma e localização, respectivamente
A Eq. 13 pode ser derivada analiticamente fornecendo a função densidade de probabilidade da GEV descrita por Blain (2011) como:

$$
\begin{aligned}
\text { FDP }=f(x) & =\frac{1}{\alpha} \times\left[1-\xi \cdot\left(\frac{x-\mu}{\alpha}\right)\right]^{\left(\frac{1+\xi}{\xi}\right)} \times \\
& \times \exp \left\{-\left[1-\xi \cdot\left(\frac{x-\mu}{\alpha}\right)\right]^{\frac{1}{\xi}}\right\}
\end{aligned}
$$

A média, a variância e o coeficiente de assimetria de uma distribuição GEV são dados, respectivamente, por:

$$
\begin{gathered}
\mathrm{E}[\mathrm{x}]=\overline{\mathrm{x}}=\mu+\frac{\alpha}{\xi} \cdot[1-\Gamma(1+\xi)] \\
\operatorname{Var}(\mathrm{x})=\left(\frac{\alpha}{\xi}\right)^{2} \cdot\left[\Gamma(1+2 \cdot \xi)-\Gamma^{2}(1+\xi)\right] \\
\gamma=\mathrm{CA}=(\text { sinal de } \xi) \cdot\left[\frac{-\Gamma(1+3 \cdot \xi)+3 \cdot \Gamma(1+\xi) \cdot \Gamma(1+2 \cdot \xi)-2 \cdot \Gamma^{3}(1+\xi)}{\left[\Gamma(1+2 \cdot \xi)-\Gamma^{2}(1+\xi)\right]^{\frac{3}{2}}}\right]
\end{gathered}
$$

Os parâmetros da Eq. 14 foram estimados pelos métodos da MV, MM e M-L. A estimativa dos parâmetros desta distribuição por MM se inicia com a Eq. 17, a qual deve ser resolvida para $\xi$, por meio de iteração numérica; em seguida se determina o valor de $\alpha$, isolando-o na Eq. 16, ou seja:

$$
\alpha=\sqrt{\frac{\xi^{2} \operatorname{Var}[\mathrm{Y}]}{\Gamma(1+2 \xi)-\Gamma^{2}(1+\xi)}}
$$

Enfim, determina-se o parâmetro de posição $\mu$, por meio da Eq. 15, chegando à seguinte expressão:

$$
\mu=E[Y]-\frac{\alpha}{\xi}[1-\Gamma(1+\xi)]
$$

Para a estimação dos parâmetros dessa distribuição por MV foram seguidas as orientações de Smith (1985), descritas em Bautista et al. (2004). Segundo este autor e supondo que há independência entre as observações, obteve-se a função de verossimilhança, sendo:

$$
\begin{aligned}
L(\theta)=L(\mu, \alpha, \xi) & =\frac{1}{\alpha^{n}} \prod_{i=1}^{n}\left\{\left[1+\xi \cdot\left(\frac{X_{i}-\mu}{\alpha}\right)\right]^{-\left(\frac{1+\xi}{\xi}\right)}\right\} \times \\
& \times \exp \left\{\sum_{i=1}^{n}\left\{-\left[1+\xi \cdot\left(\frac{x_{i}-\mu}{\alpha}\right)\right]^{-\frac{1}{\xi}}\right\}\right\}
\end{aligned}
$$

Logo, o logaritmo desta função é dado por: 


$$
\begin{aligned}
\mathrm{L}(\theta)=\ln [\mathrm{L}(\mu, \alpha, \xi)] & =\sum_{\mathrm{i}=1}^{\mathrm{n}}-\ln \alpha-\left(\frac{1+\xi}{\xi}\right) \times \\
& \times \ln \left[1+\xi\left(\frac{\mathrm{x}_{\mathrm{i}}-\mu}{\alpha}\right)\right]- \\
& -\left[1+\xi\left(\frac{\mathrm{x}_{\mathrm{i}}-\mu}{\alpha}\right)\right]^{-\frac{1}{\xi}}
\end{aligned}
$$

Os estimadores $(\mu, \alpha, \xi)$ foram obtidos pela solução do sistema de equações não-lineares formado pelas derivadas de primeira ordem da Eq. 21, em relação a cada parâmetro, igualadas a zero, isto é:

$$
\begin{gathered}
\frac{1}{\hat{\alpha}} \sum_{\mathrm{i}=1}^{\mathrm{n}}\left(\frac{1+\hat{\xi}-\mathrm{w}_{1}^{-\frac{1}{\xi}}}{\hat{\alpha}}\right)=0 \\
-\frac{\mathrm{n}}{\hat{\alpha}}-\frac{1}{\hat{\alpha}^{2}} \sum_{\mathrm{i}=1}^{\mathrm{n}}\left\{\frac{\left(\mathrm{x}_{\mathrm{i}}-\mu\right)\left[(1+\hat{\xi})-\mathrm{w}_{1}^{-\frac{1}{\hat{\xi}}}\right]}{\mathrm{w}_{1}}\right\}=0 \\
\sum_{\mathrm{i}=1}^{\mathrm{n}}\left\{\left(1-\mathrm{w}_{\mathrm{i}}^{-\frac{1}{\hat{\xi}}}\right)\left[\frac{1}{\hat{\xi}^{2}} \ln \left(\mathrm{w}_{\mathrm{i}}\right)-\frac{\left(\mathrm{x}_{\mathrm{i}}-\hat{\mu}\right)}{\hat{\xi} \hat{\alpha} \mathrm{w}_{\mathrm{i}}}\right]-\frac{\left(\mathrm{x}_{\mathrm{i}}-\hat{\mu}\right)}{\hat{\alpha} \mathrm{w}_{\mathrm{i}}}\right\}=0
\end{gathered}
$$

em que:

$$
\mathrm{w}_{\mathrm{i}}=1+\hat{\xi}\left(\frac{\mathrm{x}_{\mathrm{i}}-\hat{\mu}}{\hat{\alpha}}\right)
$$

Com vista à determinação dos parâmetros da GEV por M-L seguiram-se as orientações de Valverde et al. (2004) e Naguettini \& Pinto (2007) utilizando-se das seguintes relações matemáticas:

$$
\begin{gathered}
\hat{\xi}=7,8590 \mathrm{C}+2,9554 \mathrm{C}^{2} \\
\hat{\alpha}=\frac{\lambda_{2} \hat{\xi}}{\Gamma(1+\hat{\xi})\left(1-2^{-\hat{\xi}}\right)} \\
\hat{\mu}=\lambda_{1}-\frac{\hat{\alpha}}{\hat{\xi}}[1-\Gamma(1+\hat{\xi})] \\
\mathrm{C}=\frac{2}{\left(3+\tau_{3}\right)}-\frac{\ln (2)}{\ln (3)} \\
\tau_{3}=-3+\frac{2\left(1-3^{-\hat{\xi}}\right)}{\left(1-2^{-\hat{\xi}}\right)}
\end{gathered}
$$

em que:

$\lambda_{1}$ e $\lambda_{1}$ - momentos de posição e de escala da distribuição, respectivamente, determinados pelas Eqs. 10 e 11

$\tau_{3} \quad$ - coeficiente de assimetria da distribuição

Para verificar o grau de ajuste das distribuições empíricas aos dados de precipitação diária máxima anual, foram utilizados os testes de aderência de $\chi_{2}$ e Filliben, nos quais é calculado um coeficiente de correlação $\left(\mathrm{r}_{\text {calc }}\right)$ entre os dados observados e os dados estimados, com base no inverso da função de distribuição de probabilidades aplicada. Se os valores tabelados $\mathrm{R}$ (valores críticos), para determinado tamanho de amostra são maiores do que $\mathrm{r}_{\text {calc }}$, a hipótese de que os dados seguem respectiva distribuição é rejeitada para o nível de significância a que, para este trabalho, foi atribuído um nível de significância de 0,05 . Segundo Naghettini \& Pinto (2007) a estatística do teste de Filliben é expressa por:

$$
\begin{gathered}
r_{\text {calc }}=\frac{\sum_{i=1}^{N}\left(y_{i}-\bar{y}\right) \cdot\left(w_{1}-\bar{w}\right)}{\sqrt{\sum_{i=1}^{N}\left(y_{i}-\bar{y}\right)^{2} \cdot \sum_{i=1}^{N}\left(w_{1}-\bar{w}\right)^{2}}} \\
\bar{y}=\frac{\sum_{i-1}^{N} y_{1}}{N} \\
\bar{w}=\frac{\sum_{i=1}^{N} w_{i}}{N} \\
w_{i}=F_{y}^{-1}\left(1-q_{i}\right) \\
\frac{i-a}{N+1-2 a}
\end{gathered}
$$

em que:

$$
\begin{array}{ll}
\mathrm{y}_{\mathrm{i}} & \text { - observações } \\
\mathrm{w}_{\mathrm{i}} & \text { - inversa da função cumulativa de probabilidades } \\
\mathrm{N} & \text { - tamanho da amostra } \\
\mathrm{i} & \text { - posição ocupada pelo valor na série } \\
\mathrm{q}_{\mathrm{i}} & \text { - probabilidades empíricas ou "posições de plotagem" } \\
\mathrm{a} & \text { - parâmetro adotado em função da distribuição utilizada }
\end{array}
$$

No teste de $\chi^{2}$, a hipótese é testada comparando-se as frequências observadas e as frequências teóricas (Mello et al., 2010), através da estatística de $\chi^{2}$, a qual é:

$$
\chi_{\text {calc }}^{2}=\sum_{\mathrm{i}=1}^{\mathrm{n}} \frac{\left(\mathrm{f}_{\text {obs }}-\mathrm{f}_{\text {esp }}\right)^{2}}{\mathrm{f}_{\text {esp }}}
$$

em que:

$$
\begin{aligned}
& \mathrm{n} \quad \text { - número de classes } \\
& \mathrm{f}_{\text {obs }} \text { e } \mathrm{f}_{\text {esp }} \text { - frequências observadas e esperadas na classe } \mathrm{i} \text {, } \\
& \text { respectivamente }
\end{aligned}
$$


Para que a distribuição de probabilidades tenha aderência aos dados, o valor de $\chi_{\text {calc }}^{2}$ deve ser menor que o $\chi_{\text {tab }}^{2}$.

\section{Resultados e Discussão}

Inicialmente se apresentam, na Tabela 2, os resultados das estatísticas dos testes $\chi^{2}$ e Filliben para as distribuições de Gumbel e GEV ajustadas por MV, MM e M-L nas sub-regiões consideradas. Os resultados demonstram que em 11 ocasiões os ajustes gerados pela metodologia da MV não foram aceitos pelos testes realizados, ou seja, houve um índice de rejeição total de aproximadamente $23 \%$. O teste de Filliben foi responsável por $54,5 \%$ dessas reprovações enquanto o teste $\chi^{2}$ ficou com os outros $45.5 \%$, demonstrando menor rigor desse teste de aderência quando se trata de ajuste por esta metodologia.

Quando se comparam as distribuições de probabilidade ajustadas por MV os resultados apontam para uma eficácia maior da distribuição de Gumbel já que dos 24 testes realizados por esta metodologia, a referida distribuição foi aprovada em 22 apresentando um índice de aceitação de 91,6\%, enquanto a GEV ficou com 62,5\%. Na comparação dos resultados dos testes de aderência com as distribuições, evidencia-se que tanto o teste $\chi^{2}$ quanto o de Filliben, foram mais restritivos para a distribuição GEV, já que dos 24 testes realizados para esta distribuição houve reprovação em $33,3 \% \operatorname{com} \chi^{2}$, sendo as estações de Ibertioga, Itumirim, Macaia e São Vicente de Minas e $41,6 \%$ com o teste de Filliben, sendo as estações de Ibertioga, Macaia, Aiuruoca, Alagoa e Carrancas. Para Gumbel não houve predominância de nenhum teste ficando apenas uma reprovação para cada teste sendo a estação de São Vicente de Minas pelo teste $\chi^{2}$ e Carvalhos pelo de Filliben.

Quando se avaliam os resultados das estatísticas dos testes ajustadas por MM, eles apontam para um desempenho geral maior desta metodologia em comparação com os ajustes produzidos por MV, caso em que dos 48 testes realizados, houve 7 reprovações contabilizando 85,4\% de aceitação para MM contra $77 \%$ para MV. Nota-se, para esta metodologia, que os resultados apresentaram discrepância com relação aos testes de aderência, sendo 1 reprovação para o teste de Filliben e 6 para o teste $\chi^{2}$. Quando se comparam apenas as distribuições, observa-se similaridade nos resultados com a GEV sendo reprovada em quatro situações, sendo 3 pelo $\chi^{2}$ e uma pelo Filliben, contra um total de três reprovações da Gumbel, todos pelo $\chi^{2}$, verificando maior restrição da metodologia MM por este teste. Um fato relevante é que tais resultados apontaram para as mesmas estações quando o ajuste foi conduzido por MV, sendo Ibertioga, Itumirim, Macaia, Carvalhos, Carrancas e São Vicente.

Os resultados dos testes de aderência para as distribuições ajustadas por M-L demonstram que esta metodologia foi a que melhor se ajustou às séries estudadas considerando-se que dos 48 testes realizados, houve apenas 3 reprovações, ou seja, 93,8\% de aceitação, sendo que todas as reprovações se deram pelo teste $\chi^{2}$. Na comparação dos resultados dos testes com as respectivas distribuições ajustadas por M-L, estes apontam para uma eficácia maior da distribuição GEV, sendo este o melhor desempenho para todas as metodologias utilizadas já que dos 24 testes realizados a referida distribuição apresenta aprovação em aproximadamente $96 \%$ dos casos, enquanto que a Gumbel apresentou $91,6 \%$ de aceitação, mesmo resultado do ajuste por $\mathrm{MV}$; esses resultados corroboram os de Oliveira et al. (2014) cujos autores utilizaram a GEV ajustada por M-L em dados de PDMA na região sul de Minas Gerais e a recomendam como melhor opção para a estimativa da PDMA em diferentes tempos de retorno.

Em um contexto geral foram realizados 144 testes de aderência, dos quais 72 para cada distribuição de

Tabela 2. Resultados dos testes de aderência de $\chi^{2}$ e Filliben para cada distribuição

\begin{tabular}{|c|c|c|c|c|c|c|c|c|c|}
\hline \multirow{2}{*}{ Estação } & \multirow{2}{*}{ Distribuiçãa } & \multicolumn{4}{|c|}{$\chi^{2}$} & \multicolumn{4}{|c|}{ Filliben } \\
\hline & & MV & MM & $M-L$ & Tabelado* & MV & MM & $M-L$ & Tabelado* \\
\hline \multirow{2}{*}{ Ibituruna } & Gumbel & $3,315 \mathrm{a}$ & $3,430 \mathrm{a}$ & $6,236 \mathrm{a}$ & 9,488 & $0,9846 \mathrm{a}$ & $0,9846 \mathrm{a}$ & $0,9814 a$ & 0,9610 \\
\hline & GEV & $1,589 \mathrm{a}$ & $5,301 \mathrm{a}$ & $2,393 \mathrm{a}$ & 7,815 & $0,9800 \mathrm{a}$ & $0,9776 \mathrm{a}$ & $0,9819 a$ & 0,9695 \\
\hline \multirow{2}{*}{ Ibertioga } & Gumbel & $10,160 \mathrm{a}$ & 26,263 na & 17,347 na & 11,070 & $0,9737 \mathrm{a}$ & $0,9737 \mathrm{a}$ & $0,9737 \mathrm{a}$ & 0,9720 \\
\hline & GEV & 23,319 na & $6,989 \mathrm{a}$ & 5,935 a & 9,488 & $0,9518 \mathrm{na}$ & $0,9920 \mathrm{a}$ & $0,9916 \mathrm{a}$ & 0,9784 \\
\hline \multirow{2}{*}{ Itumirim } & Gumbel & $6,084 \mathrm{a}$ & $10,74 \mathrm{a}$ & $9,026 \mathrm{a}$ & 11,070 & 0,9778 a & $0,9778 \mathrm{a}$ & $0,9778 \mathrm{a}$ & 0,9706 \\
\hline & GEV & 13,623 na & $6,482 \mathrm{a}$ & $3,074 \mathrm{a}$ & 9,488 & $0,9852 \mathrm{a}$ & 0,9774 na & $0,9873 \mathrm{a}$ & 0,9775 \\
\hline \multirow{2}{*}{ Macaia } & Gumbel & $5,709 a$ & $9,522 \mathrm{na}$ & $7,080 \mathrm{a}$ & 7,815 & $0,9731 \mathrm{a}$ & $0,9731 \mathrm{a}$ & $0,9731 \mathrm{a}$ & 0,9553 \\
\hline & GEV & 9,045 na & $2,864 \mathrm{a}$ & $2,897 \mathrm{a}$ & 5,991 & $0,9370 \mathrm{na}$ & $0,9899 \mathrm{a}$ & $0,9896 \mathrm{a}$ & 0,9636 \\
\hline \multirow{2}{*}{ Carrancas } & Gumbel & $5,356 \mathrm{a}$ & $1,901 \mathrm{a}$ & $0,711 \mathrm{a}$ & 5,991 & $0,9593 \mathrm{a}$ & $0,9593 \mathrm{a}$ & $0,9553 \mathrm{a}$ & 0,9499 \\
\hline & GEV & $1,964 \mathrm{a}$ & 15,13 na & $0,148 \mathrm{a}$ & 3,841 & 0,9378 na & $0,9595 \mathrm{a}$ & $0,9868 \mathrm{a}$ & 0,9584 \\
\hline \multirow{4}{*}{$\begin{array}{l}\text { Madre de } \\
\text { Deus } \\
\text { Faz, } \\
\text { Laranjeiras }\end{array}$} & Gumbel & $5,007 \mathrm{a}$ & $4,868 \mathrm{a}$ & $4,609 \mathrm{a}$ & 11,070 & $0,9887 \mathrm{a}$ & $0,9887 \mathrm{a}$ & $0,9887 \mathrm{a}$ & 0,9706 \\
\hline & GEV & $4,500 \mathrm{a}$ & $4,484 \mathrm{a}$ & $4,287 \mathrm{a}$ & 9,488 & $0,9858 \mathrm{a}$ & $0,9893 \mathrm{a}$ & $0,9874 \mathrm{a}$ & 0,9775 \\
\hline & Gumbel & $1,244 \mathrm{a}$ & $1,212 \mathrm{a}$ & $1,345 \mathrm{a}$ & 7,815 & $0,9945 \mathrm{a}$ & $0,9945 \mathrm{a}$ & $0,9945 \mathrm{a}$ & 0,9553 \\
\hline & GEV & $1,712 \mathrm{a}$ & $2,395 \mathrm{a}$ & $1,344 \mathrm{a}$ & 5,991 & $0,9912 \mathrm{a}$ & $0,9942 \mathrm{a}$ & $0,9948 \mathrm{a}$ & 0,9636 \\
\hline \multirow{2}{*}{ São Vicente } & Gumbel & $12,801 \mathrm{na}$ & 11,310 na & 14,634 na & 11,070 & $0,9748 \mathrm{a}$ & $0,9748 \mathrm{a}$ & $0,9732 \mathrm{a}$ & 0,9677 \\
\hline & GEV & 12,921 na & 10,232 na & 17,115 na & 9,488 & $0,9761 \mathrm{a}$ & $0,9764 \mathrm{a}$ & $0,9775 \mathrm{a}$ & 0,9758 \\
\hline \multirow{2}{*}{ Aiuruoca } & Gumbel & $5,872 \mathrm{a}$ & $5,540 \mathrm{a}$ & $4,482 \mathrm{a}$ & 11,070 & $0,9863 \mathrm{a}$ & $0,9863 \mathrm{a}$ & $0,9886 \mathrm{a}$ & 0,9706 \\
\hline & GEV & $8,402 \mathrm{a}$ & $2,765 \mathrm{a}$ & $3,301 \mathrm{a}$ & 9,488 & 0,9494 na & $0,9895 \mathrm{a}$ & $0,9925 \mathrm{a}$ & 0,9775 \\
\hline \multirow{2}{*}{ Alagoa } & Gumbel & $2,506 \mathrm{a}$ & $3,951 \mathrm{a}$ & $2,909 a$ & 11,070 & $0,9803 \mathrm{a}$ & $0,9803 \mathrm{a}$ & $0,9803 \mathrm{a}$ & 0,9720 \\
\hline & GEV & $2,057 \mathrm{a}$ & $2,231 \mathrm{a}$ & $6,322 \mathrm{a}$ & 9,488 & $0,9680 \mathrm{na}$ & $0,9900 \mathrm{a}$ & $0,9893 \mathrm{a}$ & 0,9784 \\
\hline \multirow{2}{*}{ Carvalhos } & Gumbel & $3,266 \mathrm{a}$ & $4,069 \mathrm{a}$ & $7,670 \mathrm{a}$ & 9,488 & 0,9475 na & 0,9917 a & $0,9917 \mathrm{a}$ & 0,9615 \\
\hline & GEV & $3,782 \mathrm{a}$ & 9,973 nа & $2,426 \mathrm{a}$ & 7,815 & 0,9897 a & $0,9918 \mathrm{a}$ & $0,9911 \mathrm{a}$ & 0,9701 \\
\hline \multirow{2}{*}{ Bom Jardim } & Gumbel & $3,571 \mathrm{a}$ & $5,744 \mathrm{a}$ & $3,973 \mathrm{a}$ & 11,070 & $0,9865 \mathrm{a}$ & $0,9865 \mathrm{a}$ & $0,9865 \mathrm{a}$ & 0,9706 \\
\hline & GEV & $3,934 \mathrm{a}$ & $3,024 \mathrm{a}$ & $3,521 \mathrm{a}$ & 9,488 & $0,9782 \mathrm{a}$ & $0,9933 \mathrm{a}$ & $0,9931 \mathrm{a}$ & 0,9775 \\
\hline
\end{tabular}

*Valores extraídos de Naghettini \& Pinto (2007), adaptados da tabela original de Stendinger et al. (1993); MV - Método do máxima verossimilhança, MM - Método dos momentos, M-L Momentos-L. , a - Adequado, na - Não adequado pelos testes de aderência 
probabilidade utilizada. Assim, a GEV apresentou-se com desempenho bastante inferior, com 14 reprovações, sendo a metodologia da $\mathrm{MV}$ a menos precisa, responsável por 64\% das reprovações. O ajuste por MM apresentou 28,6\% das reprovações seguido do ajuste por M-L que apresentou o melhor desempenho para esta distribuição, sendo 3 reprovações, totalizando apenas 7,1\% de rejeição.

Os resultados dos testes para a distribuição Gumbel apontam para um desempenho superior, com 7 reprovações no total, o que representa, em termos percentuais, 90,3\% de aceitação, As metodologias de ajuste desta distribuição não influenciaram os resultados obtidos apresentando-se com desempenho bastante semelhante, variando de $57,1 \%$ para o ajuste com MM e 71,4\% para os ajustes por MV e M-L; dos 48 testes de aderência realizados para cada metodologia os resultados demonstraram melhor desempenho para a metodologia M-L com 93,8\% de aprovação, seguido do método MM que se apresenta como a segunda opção de ajuste, com aproveitamento de $85,4 \%$ e, por fim, a metodologia MV que, contrariamente ao que é descrito na literatura, apresentou o pior desempenho neste estudo, com $77,1 \%$.

Quando se avaliam as sub-regiões separadamente, observa-se que a metodologia que melhor se ajustou foi a M-L, independentemente da sub-região, a qual deve ser selecionada para o ajuste de distribuições assintóticas no Alto Rio Grande. Esses resultados corroboram os descritos em Shahzadi et al. (2013), que estudaram a frequência de precipitação máxima anual no Paquistão usando momentos-L; entretanto, quando se avalia a metodologia ajustada à distribuição tem-se o seguinte panorama: para a sub-região SM obtiveram-se os mesmos resultados com o ajuste da Gumbel M-L, Gumbel MV e GEV M-L; logo, todas podem ser selecionadas para estudos relacionados à precipitação diária máxima anual cuja seleção deve ficar a critério da simplicidade matemática que a distribuição Gumbel proporciona sendo, neste caso, a melhor opção para esta sub-região, a distribuição de Gumbel ajustada por M-L.
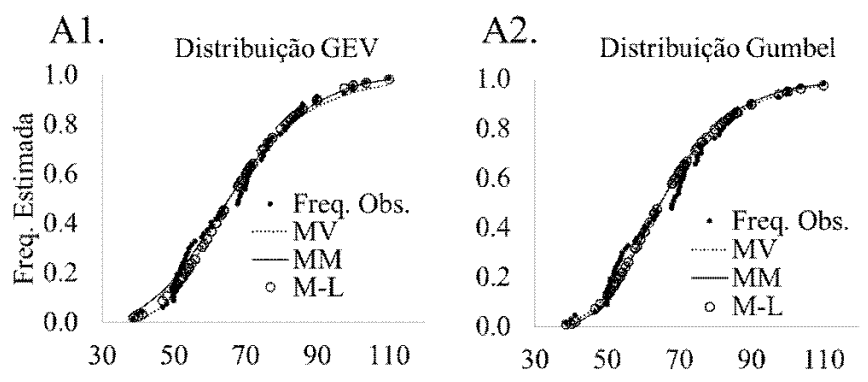

\section{C1.}

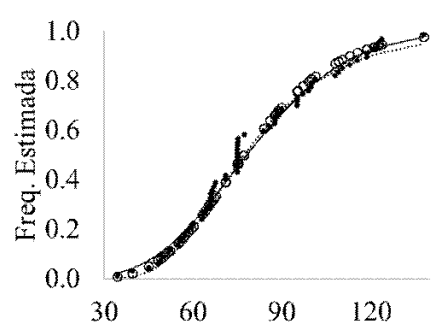

\section{C2.}

Para a sub-região PCV, os resultados se semelham aos da SM, com os melhores desempenhos da GEV-ML e Gumbel MV, com a distribuição GEV-ML devendo ser selecionada, preferencialmente, como a melhor opção para estudos desta natureza; no caso da região de transição entre os Campos das Vertentes e Serra da Mantiqueira, obtiveram-se bons resultados com a distribuição Gumbel, independente da metodologia de ajuste, bem como com a GEV ajustada por M-L; assim, a Gumbel ajustada por M-L deve ser selecionada.

Os ajustes das distribuições para as sub-regiões do Alto Rio Grande podem ser visualizados nas Figuras 1 (SM), 2 (PCV) e 3 (TR). Quando se observa a Figura 1, percebe-se boa relação de ajuste das distribuições com as metodologias empregadas, Visualmente, pode-se perceber uma pequena variação no caudal superior da Figura 1B1, que representa a estação de Aiuruoca ajustada por MV, mesmo caso da Figura 2A1 e Figura 2C1, representando Ibertioga e Macaia, ambas por MV, e por último a Figura $3 \mathrm{C} 1$, representando a estação de Carrancas ajustada pela mesma metodologia demonstrando que a metodologia MV, independente das subregiões consideradas, pode gerar incertezas na modelagem as quais são passíveis de estarem associadas às características da massa de dados utilizada, ou seja, do regime de precipitação e da gênese das chuvas intensas para cada localidade. Com isto são notórias a importância e a necessidade de realização de testes de aderência para a seleção da melhor distribuição e método de ajuste.

Outra constatação importante fica por conta do ajuste da estação de Carrancas (Figuras 2C1 e 2C2), podendo-se observar que apenas para este caso específico o tamanho da amostra de precipitação diária máxima anual refletiu negativamente no ajuste das distribuições tendo em vista que a referida estação foi a que apresentou a menor massa de dados disponível; ressalta-se, ainda, que a distribuição Gumbel se apresenta com ligeira vantagem sob a GEV.
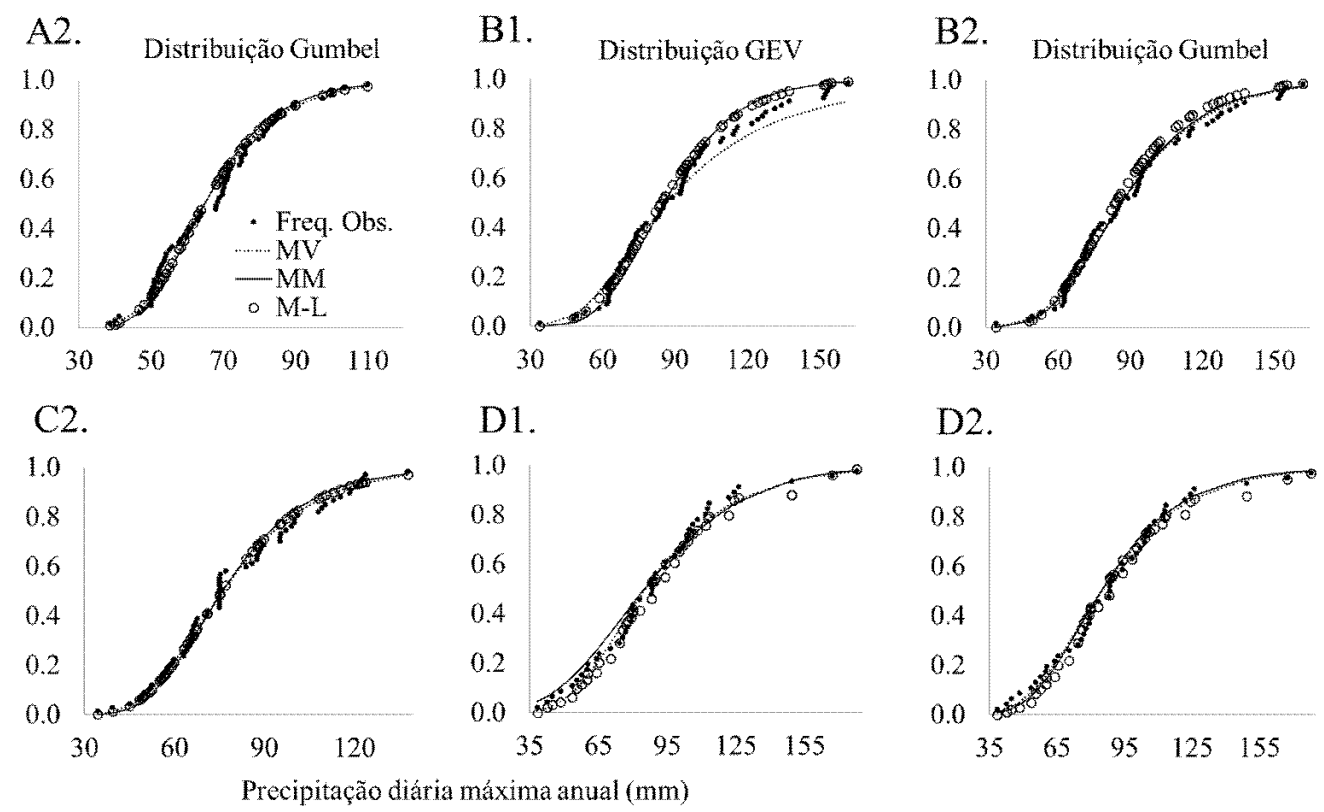

D2.

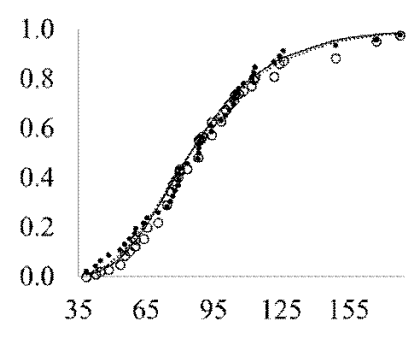

Figura 1, Ajuste para as estações da sub-região SM: A1 e A2, Bom Jardim; B1 e B2, Aiuruoca; C1 e C2, Alagoa e D1 e D2, Carvalhos 


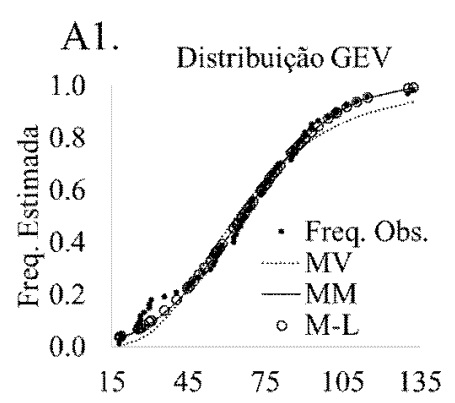

C1.

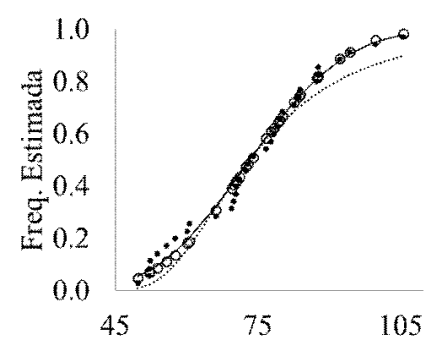

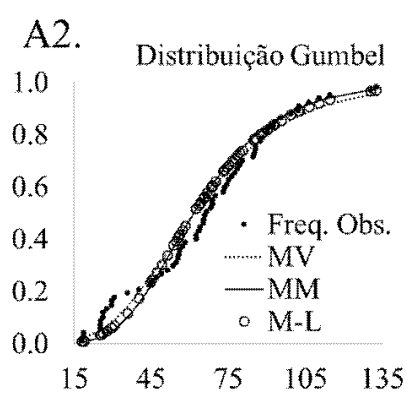

C2.

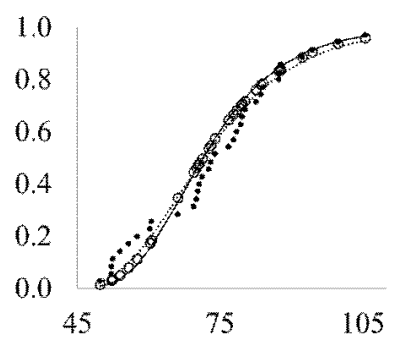

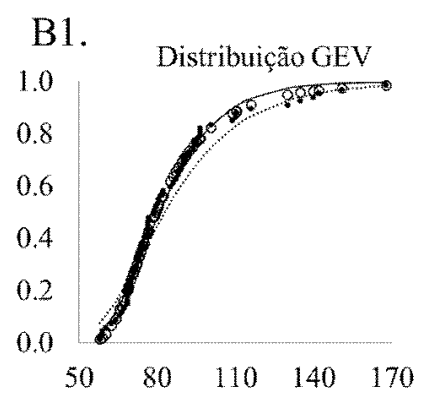

D1.

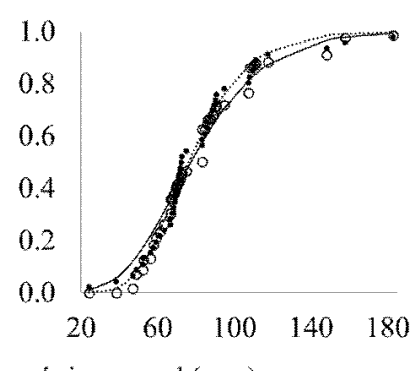

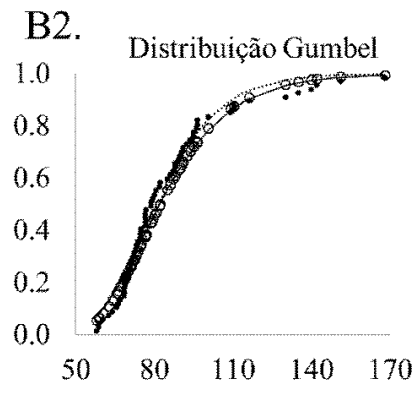

D2.

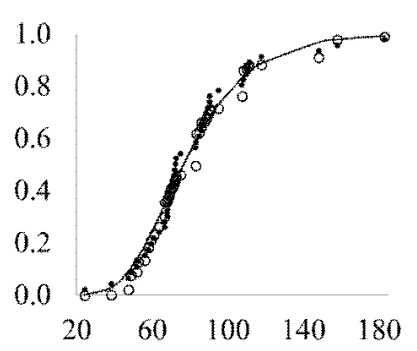

Precipitação diária máxima anual $(\mathrm{mm})$

Figura 2, Ajuste para as estações da sub-região PCV: A1 e A2, Ibertioga; B1 e B2, Itumirim; C1 e C2, Macaia e D1 e D2, Ibituruna

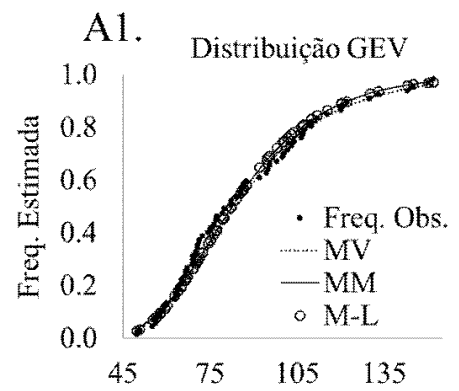

C1.

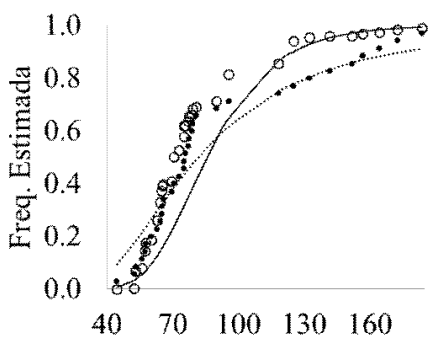

A2.

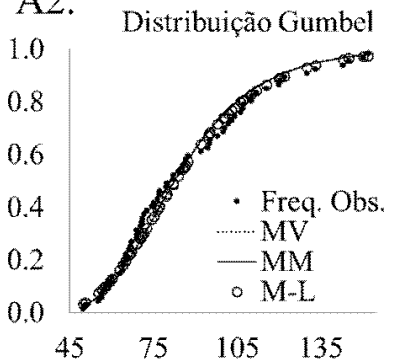

C2.

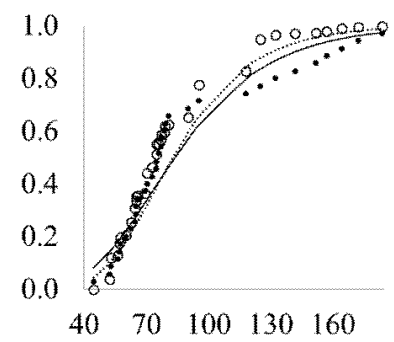

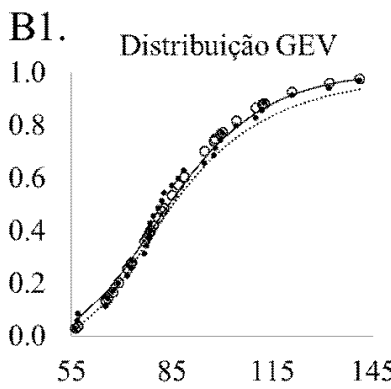

D1.

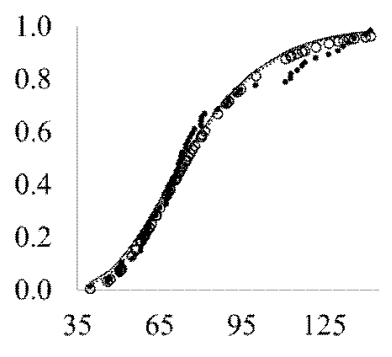

B2. Distribuição Gumbel

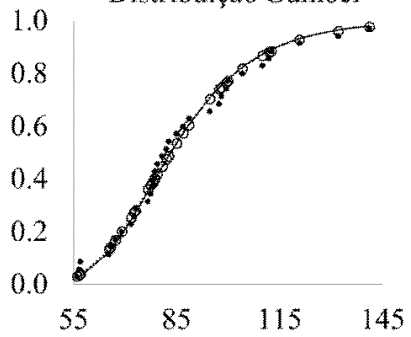

D2.

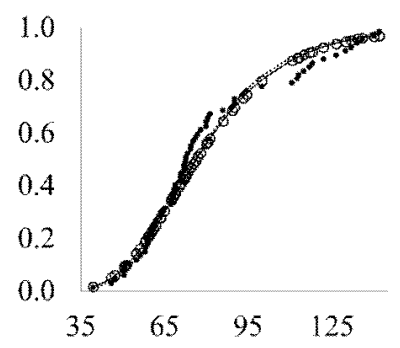

Precipitação diária máxima anual (mm)

Figura 3, Ajuste para as estações da sub-região TR: A) Madre de Deus, B) Faz, Laranjeira, C) Carrancas e D) São Vicente

\section{Conclusões}

1. Os ajustes das distribuições de probabilidades utilizadas pelas metodologias propostas foram influenciados pelas características climáticas e geográficas de cada sub-região estudada.

2. A metodologia dos momentos-L mostrou-se como a melhor opção para estudos relacionados à precipitação diária máxima anual, nas sub-regiões do Alto Rio Grande.

3. Para as sub-regiões Serra da Mantiqueira e Transição, recomenda-se o ajuste da distribuição de Gumbel enquanto para o Planalto Campos das Vertentes a melhor opção de ajuste é a distribuição GEV.

\section{Literatura Citada}

Alvarenga, C. C.; Mello, C. R. de; Mello, J. M. de; Viola, M. R. Continuidade espacial da condutividade hidráulica saturada do solo na bacia hidrográfica do Alto Rio Grande, MG. Revista Brasileira de Ciência do Solo, v.35, p.1745-1757, 2011. http:// dx.doi.org/10.1590/S0100-06832011000500029

Ávila, L. F.; Mello, C. R. de; Viola, M. R. Mapeamento da precipitação mínima provável para o sul de Minas Gerais. Revista Brasileira de Engenharia Agrícola e Ambiental, v.13. p.906-915, 2009. http://dx.doi.org/10.1590/S1415-43662009000700013

Bautista, E. A. L.; Zocchi, S. S.; Angelocci, L. R. A. Distribuição generalizada de valores extremos aplicada ao ajuste dos dados de velocidade máxima de vento em Piracicaba, São Paulo, Brasil. Revista Matemática e Estatística, v.22, p.95-111, 2004. 
Blain, G. C. Cento e vinte anos de totais extremos de precipitação pluvial máxima diária em Campinas, Estado de São Paulo: Análises estatísticas. Bragantia, v.70, p.722-728, 2011. http:// dx.doi.org/10.1590/S0006-87052011000300031

Blain, G. C.; Camargo, M. B. P. de. Probabilistic structure of an annual extreme rainfall series of a coastal area of the state of São Paulo, Brazil. Engenharia Agrícola, v.32, p.552-559, 2012. http:// dx.doi.org/10.1590/S0100-69162012000300014

Hartmann, M.; Moala, F. A.; Mendonça, M. A. Estudo das precipitações máximas anuais em Presidente Prudente. Revista Brasileira de Meteorologia, v.26, p.561-568, 2011. http://dx.doi. org/10.1590/S0102-77862011000400006

Hosking, J. R. M. L-moments: Analysis and estimation of distributions using linear combinations of order statistics. Journal of the Royal Statistical Society, v.52, p.105-124, 1990.

Mello, C. R. de; Norton, L. D.; Curi, N.; Yanagi, S. N. M. Sea surface temperature (sst) and rainfall erosivity in the upper Grande River basin, southeast Brazil. Ciência e Agrotecnologia, v.36, p.53-59, 2012. http://dx.doi.org/10.1590/S1413-70542012000100007

Mello, C. R. de; Viola, M. R.; Beskow, S. Vazões máximas e mínimas para bacias hidrográficas da região Alto Rio Grande, MG. Ciência e Agrotecnologia, v.34, p.494-502, 2010. http://dx.doi. org/10.1590/S1413-70542010000200031

Naghettini, M.; Pinto, E. J. de A. Hidrologia estatística, 1,ed, Belo Horizonte: CPRM, 2007, 552p.
Oliveira, A. S. de; Mello, C. R. de; Franco, C. S.; Marques, R. F. de P. V.; Silva, A. M. Aplicabilidade da distribuição GEV ao estudo da precipitação máxima diária anual na região sul de Minas Gerais. Revista Agrogeoambiental, v.6, p.31-44, 2014.

Shahzadi, A.; Akhter, A. S.; Saf, B. Regional frequency analysis of annual maximum rainfall in monsoon region of Pakistan using L-moments. Pakistan Journal of Statistics and Operation Research, v.9, p.111-136, 2013.

Sharda, V. N.; Das, P. K.; Ojasvi, P. R. Probability distributions of extreme rainfall series for conservation planning in a subhumid climate. American Society of Agricultural and Biological Engineers, v.24, p.447-453, 2008.

Smith, R. L. Maximum likelihood estimation in a class of non regular cases. Biometrika, v.72, p.67-92, 1985. http://dx.doi. org/10.1093/biomet/72.1.67

Stendinger, J. R.; Vogel, R.M.; Foufoulageorgiov, E. Frequency analysis of extreme events. Handbook of Hydrology, 1993, Chapter 18, p.1-18.

Takara, K.; Kobayashi, K. Frequency analysis of extreme events for disaster management. Journal of Disaster Research, v.8, p.147148, 2013.

Valverde, A. E. L.; Leite, H. G.; Silva, D. D. da; Pruski, F. F. Momentos-L: Teoria e aplicação em hidrologia. Revista Árvore, v.28, p.927-933, 2004. http://dx.doi.org/10.1590/S010067622004000600019 